\title{
Can Robots Elicit Different Comfortability Levels?
}

\author{
Maria Elena Lechuga Redondo ${ }^{1,2[0000-0001-6375-9450]}$ maria.lechuga@iit.it, \\ Alessia Vignolo ${ }^{3[0000-0002-9594-5159]}$, Radoslaw \\ Niewiadomski ${ }^{4,3[0000-0002-0476-0803]}$, Francesco Rea ${ }^{1[0000-0001-8535-223 X]}$, and \\ Alessandra Sciutti ${ }^{3[0000-0002-1056-3398]}$ \\ 1 Italian Institute of Technology - RBCS Unit \\ 2 University of Genoa - DIBRIS Department \\ 3 Italian Institute of Technology - CONTACT Unit \\ ${ }^{4}$ University of Trento - PSC Department
}

\begin{abstract}
Social interactions entail often complex and dynamic situations that follow non-explicit, unwritten rules. Comprehending those signals and knowing how to respond becomes the key to the success of any social communication. Thus, in order to integrate a robot into a social context it should be capable of (at least) understanding others' emotional states. Nonetheless, mastering such skill is beyond reach for current robotics which is why we introduce the single internal state which we believe reveals the most regarding interactive communications. We named it Comfortability and defined it as (disapproving of or approving of) the situation that arises as a result of a social interaction which influences one's own desire of maintaining or withdrawing from it.

Consequently, in this paper we aim to show that Comfortability can be evoked by robots, investigating at the same time its connection with other emotional states. To do that, we performed two online experiments on 196 participants asking them to imagine being interviewed by a reporter on a sensitive topic. The interviewer's actions were presented in two different formats: the first experiment (the Narrative Context) presented the actions as text; whereas the second experiment (the Visual Context) presented the actions as videos performed by the humanoid robot iCub. The actions were designed to evoke different Comfortability levels. According to the experimental results, Comfortability differs from the other reported emotional and affective states and more importantly, it can be evoked by both, humans and robots in an imaginary interaction.
\end{abstract}

Keywords: Social Interaction · Humanoid Robot · Affective computing

\section{Introduction}

If you had to think which aspect of an interaction is the one that matters the most to make it successful, what would you say?

It is known that mastering social intelligence is key to develop healthy relationships [4]. Thus, recognizing others' emotional states and adapting accordingly becomes crucial when talking about social communications. However, these 
states are complex phenomena $[3,11]$, which is why they are the topic of intense research not only in Psychology, but also in fields where artificial agents have to establish relationships with humans, such as Computer Science and Robotics.

Since mastering such a complex field as a whole is beyond reach for current robotics, we aim to find a simpler representation, i.e., a single internal state that goes beyond understanding if the person is engaged, revealing also the affective impact of the ongoing interaction (see [10] for a deeper discussion). Indeed, we believe it is important for a social agent to understand the way the other person is feeling regarding the action it just performed. It needs to comprehend how "comfortable/uncomfortable" the other person is, to change and adapt its behaviour accordingly. Therefore, we introduce a new internal state situated in a uni-dimensional scale from being extremely uncomfortable to extremely comfortable which we name Comfortability and define as follows: (disapproving of or approving of) the situation that arises as a result of a social interaction which influences one's own desire of maintaining or withdrawing from it.

As a representative example, Pettinati and Arkin [9] mentioned in the introduction of their paper the story of a Parkinson's patient who was "Uncomfortable" as a consequence of her caregiver's comment. The caregiver told her that she seemed depressed, whereas instead she was unable to perform non-verbal communication as usual because of the disease. The authors argued that companions (either humans or robots) should be capable of dealing with this type of situations e.g., by realizing that they did something "wrong" and proactively try to restore the interaction. They wrote "she may accept what is being presented to her, but she is not agreeable to it and does not necessarily understand why the caregiver feels this way", which is in line with the Comfortability definition provided above. Caregivers (or individuals) who cannot detect the Comfortability level of their partner cannot understand when they are offending, annoying or scaring them. This behaviour might unintentionally induce partners to abandon the interaction and more importantly, the impossibility to learn from mistakes.

For this reason, we believe it is fundamental to endow future robots (or artificial agents) with the capability of detecting human Comfortability and adapting their behaviour accordingly. In fact, our long term goal is to build that system [10], hence in this paper we address two main research questions:

- Can Comfortability capture the range of positive and negative states that can be evoked in an interaction? Is it different from any other affective state?

- Can a humanoid robot evoke different Comfortability levels on people? Would it differ from the way it is evoked in human-human interactions?

\section{State of the Art}

Comfortability is a concept that people might use on a daily basis, which has also been addressed by several HRI researchers. Nonetheless, as there is no official definition or name, the word Comfort is often used despite their difference. 
Whereas Comfort tackles both physical and psychological aspects in both noninteractive (e.g., sitting in a sofa, looking at a picture (discomfort sub-scale of RoSAS [2])) and interactive contexts (e.g., receiving a massage, having a conversation); Comfortability focuses on the psychological aspect and solely emerges in interactive scenarios (e.g., having a conversation).

An example of the research focusing on Comfort is by Sun et al. [16] who were interested in finding an ideal configuration for the RIBA robot to lift and carry hospital patients assuring their maximum "comfort and safety". They found that the robot's arms distance should be adapted to the patients body.

On the other hand, other researchers addressed Comfortability even though they might refer to it as "Comfort". In particular, Koay et al. [5] developed a handheld "Comfort Level Device" and made subjects report their own "Comfort" while performing a task in a simulated living room scenario in the presence of the PeopleBot robot. The subject had to search some books and write their titles over a whiteboard while the robot was moving around. They found that the situations in which the robot was moving behind the subjects, blocking or colliding with their path, were the ones reported as more "Uncomfortable". At the same time, Ball et al. [1] studied people's reactions when a robot is approaching. Especially, they measured the "Comfort" levels of seated pairs of people engaged in a collaborative task (solving a jigsaw puzzle) while the Adept Pioneer 3DX robot approached them from 8 different angles. Every time that the robot approached them, it asked "Please rate your comfort level regarding the robot's most recent approach path". Additionally, Schneeberger et al. [13] created a strained situation between a human and another agent (two conditions: human vs virtual agent) to explore if there is a difference in the authority they inspire. Particularly, they defined a list of tasks which were meant to be "stressful" and "shameful". Some of them were extracted from Menne [7] and others created (e.g., dance the chicken dance, get a booger out of your nose, tell me something really insulting). To verify and choose the 18 most "Uncomfortable" options they ran a questionnaire among 24 participants where quoting literally "each item had to be answered on a scale ranging from 1 (not uncomfortable) to 7 (very uncomfortable)". Similarly, Pettinati and Arkin [9] explored the impact of integrating a Robokind R25 robot in "strained" hierarchical relationships. To create the strained situation a confederate and a participant were asked to debate during 15 minutes about a recent controversial topic. During the discussion the robot was present but did not intervene. Seeking to discover the impact of an unengaged robot in strained situations, they found that its influence was minimal. Matsufuji et al.[6] developed a multi-modal system to detect "awkward" situations considering voice intonation and body posture. To create the awkward situation participants had to lie. The system acquired an accuracy of $80 \%$.

Analyzing these studies, we observed that the topic of "comfort in interactions" is central to social robotics and we got a closer idea of the behaviors that might influence it, as well as its practical applications in HRI scenarios. Notwithstanding, we believe that it is still missing a unifying definition, able to capture the multiple facets of this concept, which is why we propose Comfortability. 


\section{Research Question}

The present study aims to formalize Comfortability and assess whether a robot can elicit it in people. For that purpose, the following hypotheses are formulated:

Hypothesis 1 : It is possible to design actions in a narrative context able to evoke different Comfortability levels (displayed through a questionnaire online).

Hypothesis 2 : It is possible to design humanoid robot actions able to evoke different Comfortability levels (displayed through a visual questionnaire online).

Hypothesis 3 : Comfortability differs from any other emotional/affective state and can arise from different combinations of multiple emotions.

\section{Methods}

To address the hypotheses, a generic scenario was designed and tested through two online questionnaires: one called Narrative Context which illustrates the actions as textual sentences; and the other called Visual Context which presents the actions as videos of the humanoid robot iCub [8].

\subsection{Generic Scenario}

The main objective was to maximize the participants' emotional/affective states by creating a strained situation in which they feel personally involved $[9,13]$. To do that, the scenario was described as follows: "Imagine that you are being interviewed by a reporter who is asking you which are your thoughts regarding a sensitive topic (e.g., religion in the schools, abortion, animal industry, illegal immigration, robots vs humanity, etc) to later display the interview in the national $T V$ channel". Then, participants had to report their feelings after being presented with some actions. Regarding the Narrative Context questionnaire, there were 30 actions (15 meant to trigger positive and 15 negative Comfortability) presented through sentences (check Table 1). In relation to the Visual Context questionnaire, there were 10 actions ( 5 meant to trigger positive and 5 negative Comfortability) which were picked from the first experiment as they were reported as the most effective in triggering the desired responses (i.e., they were the closest to the Comfortability scale extremes). This time, the questions were presented through videos of iCub saying the sentences while changing its facial expressions and moving its torso, arms and head accordingly (See Fig.1, and https://youtu.be/QXPSVnbvgJM).

The actions and robot's movements were defined from zero, taking inspiration from [14] and [12] respectively. To remove any dependencies between actions, participants were told "please think of them as independent events which are not connected between each other". 
Table 1. The 10 selected actions for the Visual Context questionnaire, where A1-A15 were meant to trigger negative Comfortability and A18-27 positive Comfortability.

\begin{tabular}{|l|l|}
\hline A1 & $\begin{array}{l}\text { After greeting the interviewer, the reporter says "Where did you get this } \\
\text { outfit? Somehow it doesn't really fit you [14]" }\end{array}$ \\
\hline A2 & $\begin{array}{l}\text { In the middle of the interview the reporter says: "Sorry that I ask, but have } \\
\text { you farted? It smells really bad here" }\end{array}$ \\
\hline A3 & $\begin{array}{l}\text { At the end of the interview, the reporter says: "Now that I know what your } \\
\text { opinions are, allow me to ask the last question of the interview. Are } \\
\text { you proud of yourself having those stupid values and ideals?" }\end{array}$ \\
\hline A14 & $\begin{array}{l}\text { In the middle of the interview, the reporter says, "why I am even doing } \\
\text { this job? I hate it! I definitely do not like people" }\end{array}$ \\
\hline A15 & $\begin{array}{l}\text { The reporter says "Buffalo buffalo Buffalo buffalo buffalo buffalo Buf- } \\
\text { falo buffalo" without any meaning behind }\end{array}$ \\
\hline A18 & $\begin{array}{l}\text { At the beginning of the interview, the reporter says, "Hello, thank you very } \\
\text { much for participating! I am very happy that you are here!" }\end{array}$ \\
\hline A19 & $\begin{array}{l}\text { At the end of the interview, the reporter says: "Thank you very much for } \\
\text { your time, I wish you have a wonderful day" }\end{array}$ \\
\hline A20 & $\begin{array}{l}\text { At the end of the interview, the reporter says: "I am very grateful for } \\
\text { this debate. Constructive conversations like this one are not usually } \\
\text { found. Thank you very much for your time!" }\end{array}$ \\
\hline A26 & $\begin{array}{l}\text { After you have presented your idea, the reporter says: "I have never thought } \\
\text { about this topic from this perspective. I am sure that it will bring } \\
\text { novel and useful information for our viewers" }\end{array}$ \\
\hline A27 & $\begin{array}{l}\text { After you have presented your idea, the reporter says: "Wow. I think you } \\
\text { are really cool." }\end{array}$ \\
\hline
\end{tabular}

\subsection{Internal States}

To indicate their feelings, participants were asked to score a list of internal states: Pride, Gratitude, Admiration, Shame, Reproach and Anger which we refer to as Emotions and Comfortability, Engagement and Confidence which we refer to as Affective States.

The Emotions were included to discover if Comfortability is equivalent to any other emotional state (hypothesis 3). Specifically, those were chosen as they were reported by the Ortony, Clore \& Collins (OCC) model [15] to be caused by other agents, which resembles Comfortability's definition. The Affective States were picked to act like attention-grabbers, given that the scoring method for the Emotions and the Affective States was different (as Comfortability is bipolar whereas discrete emotions are not). To report the Emotions, participants were asked: "Please, indicate how strongly you would feel the following internal states from Not at all to Extremely" and then, they were presented with a Five-Likert Scale (Not at all, A little, Moderately, Quite a bit and Extremely). To report the Affective States, they were asked: "Please, indicate how strongly you would feel the following internal states from negative to positive" and then, they were

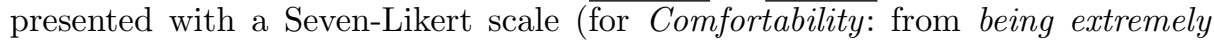




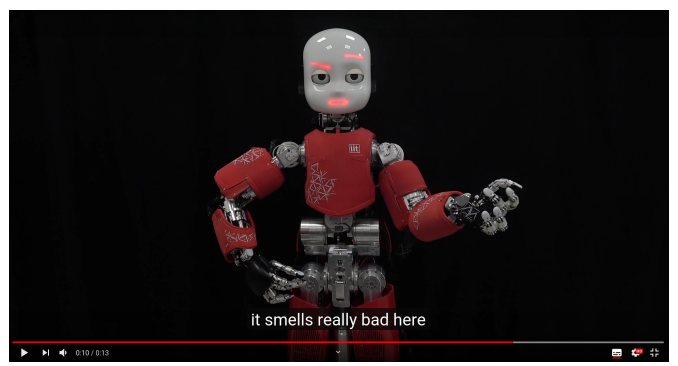

Fig. 1. Robot iCub performing one of the actions for the Visual Context questionnaire.

(uncomfortable to comfortable); for Engagement from being extremely (disengaged to engaged); for Confidence: from being extremely (insecure to confident)).

\subsection{Online Questionnaires}

Both questionnaires were submitted through the Amazon Mechanical Turk platform where random naive participants were recruited. To assure reliable data two attention check questions were added : 1) Ignore the previous sentence and future indications and mark the third column for all the rows in this page;2) Please indicate the context that matches better for the story that you were asked to imagine in the survey (There are cameras and microphones/I am in the wild surrounded by animals/ The music is amazing/I definitely have to go back to this concert/ It was a bad experience as the quality of the food was quite poor).

For the Narrative Context questionnaire, 155 participants were tested in which 55 failed at least one of the attention check questions and therefore were discarded, leaving a total of $\mathbf{1 0 0}$ participants (60\% male and $40 \%$ female). They were paid 2.15 dollars and they completed the task in 19.2 minutes on average.

For the Visual Context questionnaire, 155 participants were tested for which 59 failed at least one of the attention check questions and therefore were excluded, leaving a total of 96 participants (64.58\% male and 35.42\% female). They were paid 1 dollar and they completed the task in 12.2 minutes on average.

\section{Results}

\section{$5.1 \quad$ Hypothesis 1}

The first step was to test the Hypothesis 1 for which the Narrative Context questionnaire was used. The results can be seen in Fig.2, where A1 to A15 (set1) includes the actions meant to trigger negative Comfortability and A16 to A30 (set2) includes the actions meant to trigger positive Comfortability. From the chart, it can be seen how indeed the reported Comfortability level for set1 is comprehended between being Extremely Uncomfortable (Intensity =1) and Neither 
(Intensity $=4$ ); and the reported Comfortability level for set2 is comprehended between Neither and being Extremely Comfortable (Intensity $=7$ ).

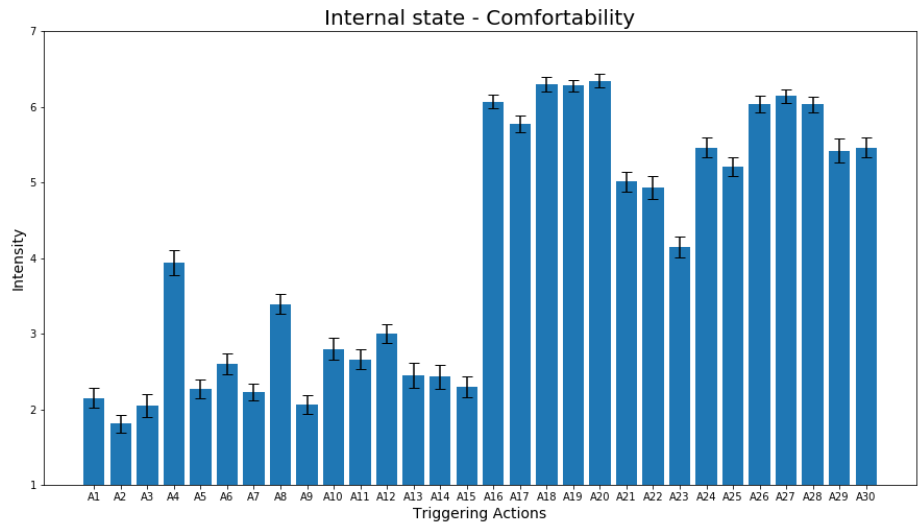

Fig. 2. All the Actions presented in the Narrative Context questionnaire.

There were some questions (A4 and A23) whose intensity, while remaining between the boundaries, was less extreme than predicted (e.g., A4:After you have presented your idea, the reporter says: "Remember that we are on a national TV channel, the arguments you present have to be based on logic and reasoning").

A non-parametric Wilcoxon Test confirmed the significant difference in median rating between $\operatorname{set} 1(M=2.54, S D=1.48)$ and $\operatorname{set} 2(M=5.64, S D=1.32)$ $(t=8833, p<.001)$, which means it is possible to design actions that evoke opposite Comfortability levels in a narrative context (which supports Hypothesis 1).

At the same time, the Affective States were compared between each other applying a Friedman's test which showed a significant difference between the Comfortability, Engagement and Confidence means' levels $(\chi(2)=396.464, p<$ .001). A Wilcoxon signed-rank post-hoc analysis confirmed that Comfortability differs from Engagement $(t=218297, p<.001)$ and Confidence $(t=139422$, $p<.001)$, after applying a Bonferroni correction. If the analysis is applied to set1 or set2 independently, similar results are obtained after applying another Friedman test $($ set1: $\chi(2)=102.213, p<.001 ;$ set2: $\chi(2)=359.991, p<.001)$. The significant difference among the median ratings of the three affective states suggests that they represent three distinct, though related aspects.

\section{$5.2 \quad$ Hypothesis 2}

Subsequently, the five actions reported as most Extremely Uncomfortable (vset1) and the five actions reported as most Extremely Comfortable (vset2) were chosen for the Visual Context questionnaire (check Table 1). 
From Fig.3, it can be noticed that the questions presented as negative and positive triggers were not only effectively distinguished in the narrative context, but also when they were acted by the humanoid robot. A Wilcoxon test confirmed the significant difference between vset1 $(M=2.49, S D=1.60)$ and $\operatorname{vset} 2(M=$ 5.70, $S D=1.23)(t=365, p<.001)$ supporting Hypothesis 2.

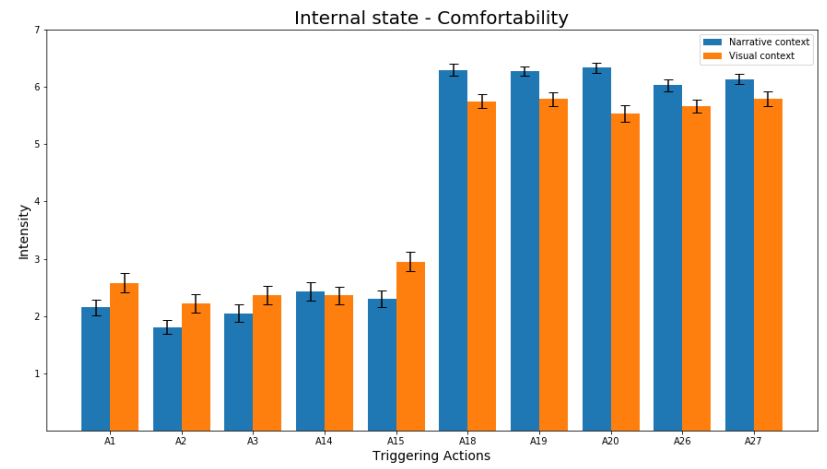

Fig. 3. Actions chosen for the Visual Context questionnaire, listed in Table 1

The Affective States were compared applying a Friedman's test, which proved that indeed, there is a significant difference among them $(\chi(2)=35.579, p<$ .001). A Post-hoc analysis including a Wilcoxon signed-rank test with a Bonferroni correction showed that, Comfortability differs from Engagement $(t=28114$, $p<.001)$ and Confidence $(t=27826, p<.001)$ similar to the Narrative Context.

Additionally, Fig.3 shows that the Comfortability values for both contexts follow a similar pattern. A mixed ANOVA with the reported Comfortability as the dependent variable, the context (narrative vs. visual) as the between factor and the designed impact (negative vs. positive triggers) as the within factor was computed. The results announced a significant effect on the designed impact $(F(1,194)=1297, p<.001)$, no effect on the context $(F(1,194)=0.654, p=$ $.42)$, and a significant interaction between the two factors $(F(1,194)=17.889$, $p<.001)$. Bonferroni corrected post-hoc tests confirmed that the ratings for the positive designed impact are significantly higher than for the negative designed impact for both experiments: the Narrative Context (considering solely the 10 selected actions; set $1: \mathrm{M}=6.22, \mathrm{SD}=0.95$ vs. set2: $\mathrm{M}=2.15, \mathrm{SD}=1.42$, with $t(99)=31.203, p<.001)$ and the $\underline{\text { Visual Context }}$ (vset1 $: \mathrm{M}=5.71, \mathrm{SD}=1.23 \mathrm{vs}$. vset2: $\mathrm{M}=2.49, \mathrm{SD}=1.60$, with $t(95)=20.584, p<.005)$. Next, the ratings for the positive designed impact are significantly higher in the Narrative Context than in the Visual Context $(\mathrm{M}=6.22, \mathrm{SD}=0.95$ vs. $\mathrm{M}=5.71, \mathrm{SD}=1.23$, with $t(169.921)=4.025, p<.001)$; and contrarily, the ratings for the negative designed impact are marginally lower in the Narrative Context than in the Visual Context 
$(\mathrm{M}=2.15, \mathrm{SD}=1.42$ vs. $\mathrm{M}=2.49, \mathrm{SD}=1.60)$, although the difference is not significant after a Bonferroni correction $(t(180.780)=-2.20, p=.058)$. This indicated that the robot videos tended to trigger less extreme Comfortability reactions than the textual sentences shown in the narrative condition.

\subsection{Hypothesis 3}

To investigate the relationship between Comfortability and the Emotional states previously introduced, several charts were plotted.

Fig. 4 shows the average Emotion value linked to the specific Comfortability level reported in both experiments. It is visible (in both contexts) that when Comfortability is reported as 1, 2 or 3 the negative Emotions' (Shame, Reproach and Anger) intensity is high whereas the positive Emotions' (Gratitude, Admiration and Pride) intensity is low. On the contrary, when Comfortability is 5,6 or 7 the tendency is the opposite. This pattern implies that as expected, the more Extremely Uncomfortable someone feels, the higher the negative Emotions' intensity will be; and similarly, if someone feels Extremely Comfortable, the positive Emotions will be high as well.

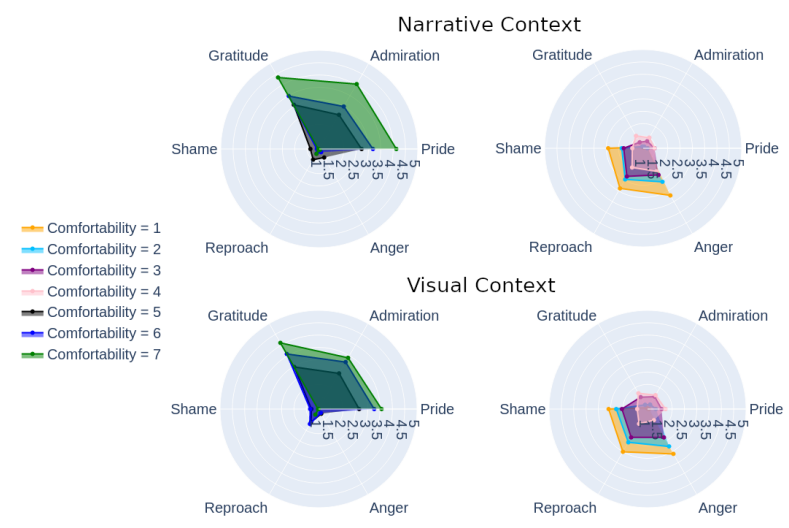

Fig. 4. Relation between the reported Emotions' and Comfortability levels.

To further investigate this pattern, making focus on the Visual Context, we computed two squared sums of the ratings associated to both sets of positive and negative Emotions for each participant and question. As a result, a set of linear regressions between this measurement of "Emotions' intensity" and Comfortability proved to be significant (Negative Emotions: slope : -.528 , $r:-.815$ and $p<.001$; Positive Emotions: slope $: .813, r: .939, p<.001$, see Fig.5).

To assess whether the same Comfortability level could be associated to different combinations of emotions, we mapped the Comfortability level in function of the three positive and negative Emotions for each action of the Visual Context 

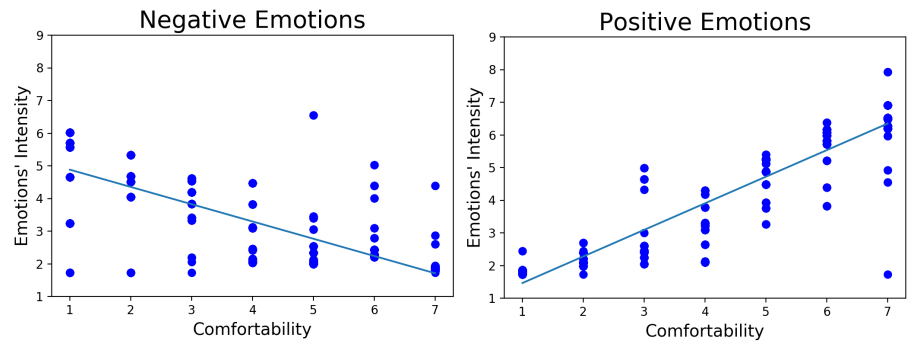

Fig. 5. Combined Emotions' ratings as a function of Comfortability's intensity.

questionnaire (see Fig.6). Each action is represented by a marker, whose shape and color depends on its Comfortability value. Color brightness reflects the combined emotions intensity (i.e., brighter symbols means being further away from the origin axis); the marker coordinates correspond to the ratings of the three reported negative or positive Emotions; and the tiny numbers correspond to the associated action listed in Table.1.
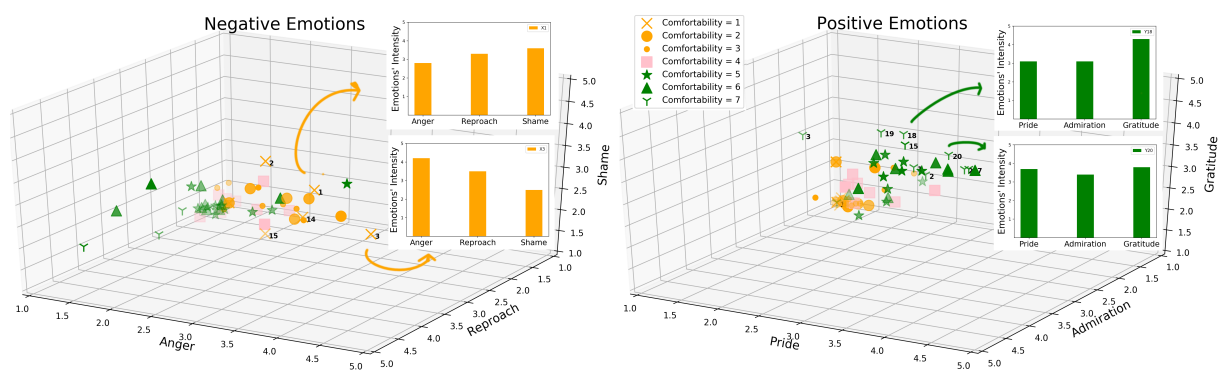

Fig. 6. Mapping between Comfortability and the three Emotions of each set, for each action regarding the Visual Context questionnaire.

Observing the Negative Emotions chart, most of the reported actions with an associated Comfortability level lower than 4 are spread over the axes. Indeed, different combinations of emotions can lead to the same Comfortability value. For example, when Comfortability is 1, some actions present Anger as the predominant Emotion (e.g., $X_{3}$ ) whereas conversely, for others Shame prevails (e.g., $X_{2}$ ). Interpreting the Positive Emotions chart, a similar tendency is appreciated. That is to say, given the same Comfortability rating (e.g., 7) there are actions in which the outstanding Emotion is Gratitude (e.g., $\left.Y_{18}\right)$ and others in which Pride dominates instead (e.g., $Y_{20}$ ). Consequently, these results show that the same Comfortability levels can be triggered alongside different sets of Emotions. Thus, Comfortability is not directly associated to any single Emotion tested here, providing evidence in favor of Hypothesis 3. 


\section{Discussion}

The aim of this paper was to investigate Comfortability, its link with Emotions and its applicability to actions performed by a robot. More in detail, we provided evidence in favor of the three hypotheses introduced in the Section 3.

First of all, we demonstrated that the actions designed as positive or negative Comfortability triggers were indeed judged as so (Hypothesis 1). More importantly, this was also confirmed when the actions were enacted by the humanoid robot, suggesting that Comfortability can be associated to robot behaviors as well (Hypothesis 2). Additionally, we noticed that the ratings related to the Visual Context were relatively less "extreme" than those obtained for the same actions depicted in the Narrative Context (see Fig.3). This might be due to the different nature of the interviewer (human or robot), but also to the different experimental context. Being free to imagine the entire interaction might have induced a stronger immersion in the situation and hence stronger ratings. Finally, it was shown that Comfortability differs from other internal states and can arise from different combinations of multiple emotions (Hypothesis 3). These results suggest that Comfortability could represent a useful uni-dimensional value reflecting the "comfort in interaction" independently of what specific emotion affected it, both in human-human and in human-robot contexts.

Still, there were some limitations that should be mentioned. On the one hand, participants were randomly collected and therefore their cultural background and familiarity with robots were not considered. Then, regarding the experimental design, there was not "an imaginary robot" in the Narrative context; neither a "human interviewer" in the Visual context, which made impossible a direct comparison. Last, the study was based on imagined stories and explicit ratings, thus Comfortability might be perceived differently in a real-live interaction.

This paper presents solely the first step towards our long-term goal of creating robots sensing and adapting to the partner's Comfortability levels. The next phase will entail a live human-robot interaction experiment, where the robot iCub will replicate the actions described here, interviewing physically a human partner. This will allow to test how Comfortability is impacted by robot actions in presence; and to measure the natural reactions and behaviors they cause. This will pave the way to the development of Comfortability detection systems in human-robot social interactive communications.

Acknowledgements. Alessandra Sciutti is supported by a Starting Grant from the European Research Council (ERC) under the European Union's Horizon 2020 research and innovation program. G.A. No. 804388, wHiSPER.

\section{References}

1. Ball, A., Silvera-Tawil, D., Rye, D., Velonaki, M.: Group comfortability when a robot approaches. In: International Conference on Social Robotics. pp. 44-53. Springer (2014) 
2. Carpinella, C.M., Wyman, A.B., Perez, M.A., Stroessner, S.J.: The robotic social attributes scale (rosas) development and validation. In: Proceedings of the 2017 ACM/IEEE International Conference on human-robot interaction. pp. 254-262 (2017)

3. Ekman, P.: Facial expression and emotion. American psychologist 48(4), 384 (1993)

4. Golleman, D.: Social intelligence: The revolutionary new science of human relationships p. 544 (2006)

5. Koay, K.L., Walters, M.L., Dautenhahn, K.: Methodological issues using a comfort level device in human-robot interactions. In: ROMAN 2005. IEEE International Workshop on Robot and Human Interactive Communication, 2005. pp. 359-364. IEEE (2005)

6. Matsufuji, A., Shiozawa, T., Hsieh, W.F., Sato-Shimokawara, E., Yamaguchi, T., Chen, L.H.: The analysis of nonverbal behavior for detecting awkward situation in communication. In: 2017 Conference on Technologies and Applications of Artificial Intelligence (TAAI). pp. 118-123. IEEE (2017)

7. Menne, I.M.: Yes, of course? an investigation on obedience and feelings of shame towards a robot. In: International Conference on Social Robotics. pp. 365-374. Springer (2017)

8. Metta, G., Sandini, G., Vernon, D., Natale, L., Nori, F.: The icub humanoid robot: an open platform for research in embodied cognition. In: Proceedings of the 8th workshop on performance metrics for intelligent systems. pp. 50-56 (2008)

9. Pettinati, M.J., Arkin, R.C.: Identifying opportunities for relationship-focused robotic interventions in strained hierarchical relationships*. In: IROS. pp. 297304 (2019)

10. Redondo, M.E.L.: Comfortability detection for adaptive human-robot interactions. In: 2019 8th International Conference on Affective Computing and Intelligent Interaction Workshops and Demos (ACIIW). pp. 35-39. IEEE (2019)

11. Russell, J.A.: A circumplex model of affect. Journal of personality and social psychology 39(6), 1161 (1980)

12. Samrose, S., Chu, W., He, C., Gao, Y., Shahrin, S.S., Bai, Z., Hoque, M.E.: Visual cues for disrespectful conversation analysis. In: 2019 8th International Conference on Affective Computing and Intelligent Interaction (ACII). pp. 580-586. IEEE (2019)

13. Schneeberger, T., Ehrhardt, S., Anglet, M.S., Gebhard, P.: Would you follow my instructions if i was not human? examining obedience towards virtual agents. In: 2019 8th International Conference on Affective Computing and Intelligent Interaction (ACII). pp. 1-7. IEEE (2019)

14. Schneeberger, T., Scholtes, M., Hilpert, B., Langer, M., Gebhard, P.: Can social agents elicit shame as humans do? In: 2019 8th International Conference on Affective Computing and Intelligent Interaction (ACII). pp. 164-170. IEEE (2019)

15. Steunebrink, B.R., Dastani, M., Meyer, J.J.C.: The occ model revisited. In: Proc. of the 4th Workshop on Emotion and Computing. Association for the Advancement of Artificial Intelligence (2009)

16. Sun, M., Nakashima, H., Hirano, S., Matsuo, K., Ding, M., Mukai, T., Qin, G., et al.: Adaptive user-centered design for safety and comfort of physical human nursing-care robot interaction. In: International Conference on Digital Human Modeling and Applications in Health, Safety, Ergonomics and Risk Management. pp. 365-372. Springer (2013) 